\title{
Room-Temperature Electroluminescence from Metal-Oxide-Silicon-Tunneling Diodes on (110) Substrates
}

\author{
Chee-Wee LIU*1, Min-Hung LEE, Shu-Tong CHANG, Miin-Jang CHEN ${ }^{* 2}$ and Ching-Fuh LIN ${ }^{* 2}$ \\ Department of Electrical Engineering, National Taiwan University, Taipei, Taiwan, R.O.C.
}

(Received August 4, 2000; accepted for publication August 21, 2000)

\begin{abstract}
We report the band-edge electroluminescence at room temperature from metal-oxide-silicon tunneling diodes on (110) substrates. An electron-hole plasma recombination model can be used to fit the emission line shape. The reliability of this electroluminescence is studied and the emission intensity varies within $10 \%$ during a $2.5 \times 10^{4} \mathrm{C} / \mathrm{cm}^{2}$ stress. A comprehensive illustration composed of localized holes, phonons, and interface roughness is given to describe the radiative process. The picture can be used to explain the enhanced electroluminescence intensity, as compared to photoluminescence, and can be used to understand the substrate orientation effect on electroluminescence intensity.
\end{abstract}

KEYWORDS: MOS diode, electroluminescence, electron-hole plasma recombination, tunneling, infrared

The band-edge electroluminescence (EL) from a metaloxide-silicon (MOS) tunneling diode attracts great attention not only due to its technological importance for possible optical interconnect applications, ${ }^{1-3)}$ but also due to its role in facilitating understanding of the radiative recombination process. ${ }^{4,5)}$ An electron-hole-plasma (EHP) recombination model was given to fit the line shape of the electroluminescence, and the band-edge luminescence nature was confirmed $^{4)}$ on the (100) substrate. The temperature-dependence study of EL spectra on (100) samples suggests that the emission lines are phonon replicas. ${ }^{5)}$ The interface roughness/surface roughness can also enhance the band-edge emission. ${ }^{2)}$ To further probe the radiation process, MOS tunneling diodes were fabricated on p-type (110) Si substrates. A comprehensive illustration is proposed to understand the effect of substrate orientation on the radiation process as well as the roles of phonons, roughness, and localized holes. Note that the natural cleavage plane on (110) $\mathrm{Si}$ is perpendicular to the substrate, hence a cavity structure can be easily fabricated. A previous study of $\mathrm{Si} / \mathrm{SiGe} / \mathrm{Si}$ quantum wells on (110) Si substrates $^{6)}$ showed that the no-phonon (NP) peak from the (110) substrate is relatively intense, as compared to the (100) substrate, probably due to the larger conduction band offset on the (110) substrate than on the (100) substrate.

The ultrathin gate oxide of the MOS tunneling diode used in this study is grown by rapid thermal oxidation (RTO) at $1000^{\circ} \mathrm{C}$. The gas flows are $500 \mathrm{sccm}$ nitrogen and $500 \mathrm{sccm}$ oxygen at a pressure of 250 mbar. Before oxidation, the sample was cleaned by HF dipping. An in situ hydrogen bake at $1000^{\circ} \mathrm{C}$ for $1 \mathrm{~min}$ was performed before the growth by RTO. The temperature was measured using a pyrometer with a close-loop control. After the growth of the ultrathin oxide, the sample was subsequently in situ annealed in hydrogen and in nitrogen for $10 \mathrm{~min}$ each at $900^{\circ} \mathrm{C}$. The nominal thickness of the oxide is about $2.4 \mathrm{~nm}$, as measured by ellipsometry. The resistivity of the $100 \mathrm{~mm}$ p-type (110) wafers is $1-10 \Omega-\mathrm{cm}$. The $\mathrm{N}$ (n-channel) MOS diodes had Al gate electrodes with circular areas defined by photolithography.

Figure 1 shows current-voltage (I-V) curves of an $\mathrm{Al} / 2.4 \mathrm{~nm}$ oxide/p-silicon diode with a circular area of

\footnotetext{
${ }^{* 1}$ E-mail address: chee@cc.ee.ntu.edu.tw Also with Graduate Institute of Electronic Engineering.

${ }^{* 2}$ Also with Institute of Electro-Optical Engineering.
}

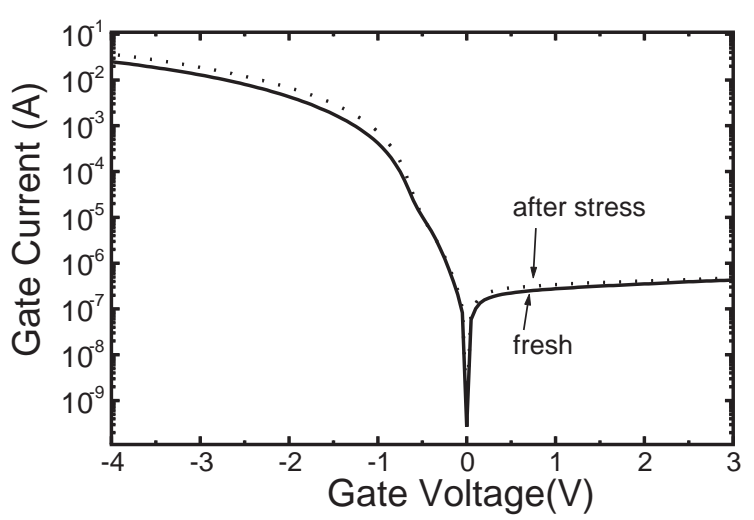

Fig. 1. Current-voltage characteristics of the MOS diode on the p-type (110) substrate before and after stress application. The stress condition is $100 \mathrm{~mA}$ for 10000 -s. The diode has an area of $4 \times 10^{-2} \mathrm{~cm}^{2}$ with an oxide thickness of $2.4 \mathrm{~nm}$.

$4 \times 10^{-2} \mathrm{~cm}^{2}$ at room temperature. For the $\mathrm{Al}$ gate under a negative bias, the electron in the Al tunnels to the $\mathrm{Si}$ substrate through the thin oxide. At a negative gate voltage lower than the flat band voltage, the electron tunnels to the interface states at the oxide/Si interface, ${ }^{7)}$ and does not contribute to the light emission process (Fig. 2). At a negative gate bias higher than the flat band voltage, the electron tunnels into the conduction band of Si substrates and recombines with the holes in the accumulation layer to emit light, as shown in Fig. 2. The kink at $\sim-0.7 \mathrm{~V}$ in the I-V curve (Fig. 1) of an NMOS device indicates the transition of these two current-transport mechanisms. Due to the large current and high resistivity of $\mathrm{Si}$ substrates $(1-10 \Omega-\mathrm{cm})$, the series resistance affects the I-V curve at a large negative gate bias $(<-1.5 \mathrm{~V})$.

Figure 3 shows the EL spectra of the MOS diode with the size of $4 \times 10^{-2} \mathrm{~cm}^{2}$ under different gate biases. The emission intensity increases as the gate bias (gate current) increases. The width of the line shape also increases with increasing gate current due to the band filling effect. The theoretical line shapes obtained from the EHP model can fit the data (Fig. 3). Details of the EHP model can be found in ref. 4. The line shape has an asymmetrical broadening. The half-width at the high-energy part of the line shape is wider, and increases more rapidly as the injection current increases, in comparison with that at the low-energy part. The device was biased at $100 \mathrm{~mA}$ for 10000-s to measure the reliability of light emission. Both 


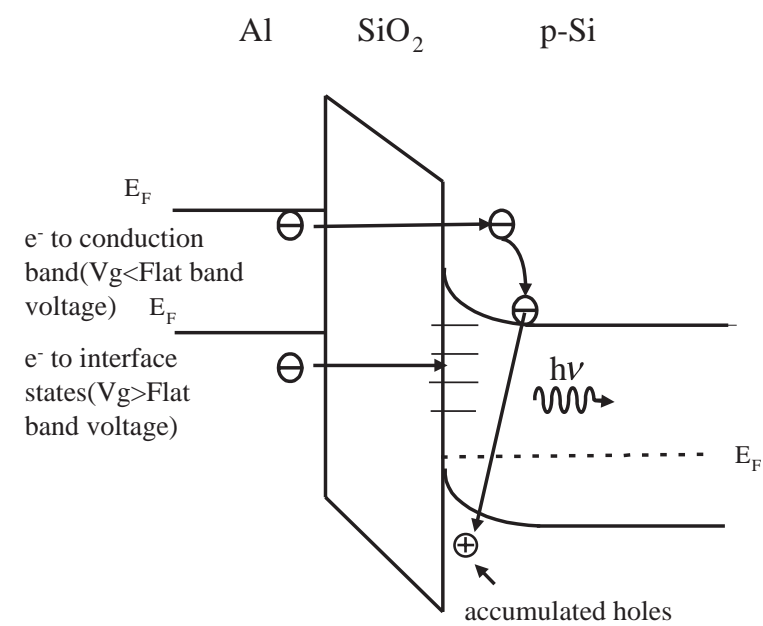

Fig. 2. Two conduction paths for the NMOS tunneling diode at a negative gate bias. The electron tunneling into the interface states does not contribute to the light-emission process.

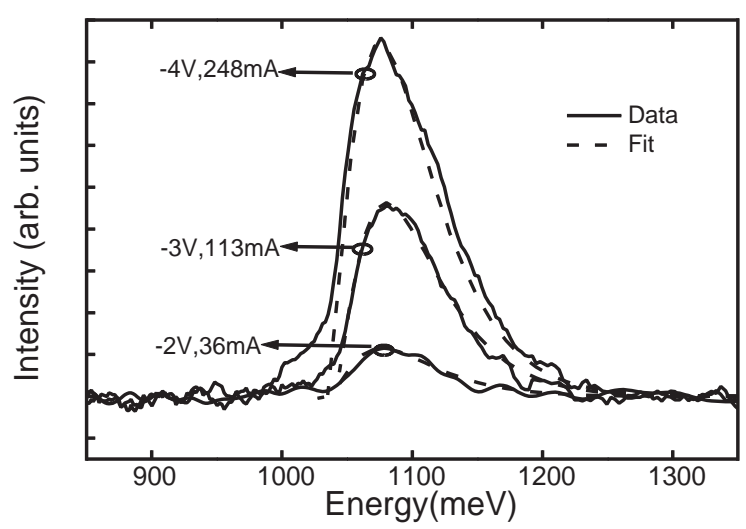

Fig. 3. EL spectra of a (110) MOS diode at a different negative gate bias. The line shape can be fitted by the electron-hole-plasma recombination model.

the voltage and light intensity change slightly within $10 \%$ during the $10000 \mathrm{~s}$ test period and most sudden decrease in light intensity occurs in the initial $1600 \mathrm{~s}$. The light intensity has a sudden rise when the voltage has a sudden decrease at $1600 \mathrm{~s}$ (Fig. 4). The sudden decrease in voltage indicates that the charge of the MOS capacitance (accumulated holes in the oxide/p-Si interface) must decrease. To reduce the concentration of the accumulated holes, the holes have to recombine with electrons and light is emitted. Therefore, the light emission intensity increases at the same time when the voltage suddenly decreases. The I-V after the 10000-s drive at $100 \mathrm{~mA}\left(2.5 \times 10^{4} \mathrm{C} / \mathrm{cm}^{2}\right.$ fluence $)$ is also shown in Fig. 1, and the large deviation from the fresh $\mathrm{I}-\mathrm{V}$ curve indicates that a soft breakdown has occurred. The soft breakdown occurs at $1600 \mathrm{~s}$ with a fluence of $4 \times 10^{3} \mathrm{C} / \mathrm{cm}^{2}$, when the voltage decreases suddenly. The fluence to breakdown is much larger than the reported data of $\sim 5 \mathrm{~A} / \mathrm{cm}^{2}$ for a 5.8 -nm-thick oxide layer. ${ }^{8)}$ This may be due to the ultra-thin oxide used in this study $(\sim 2.4 \mathrm{~nm})$.

To understand the effect of substrate orientation on electroluminescence, we present a illustration of the radiative process composed of TO phonons, interface roughness, and confined holes in the accumulation region (Fig. 5). The hole con-

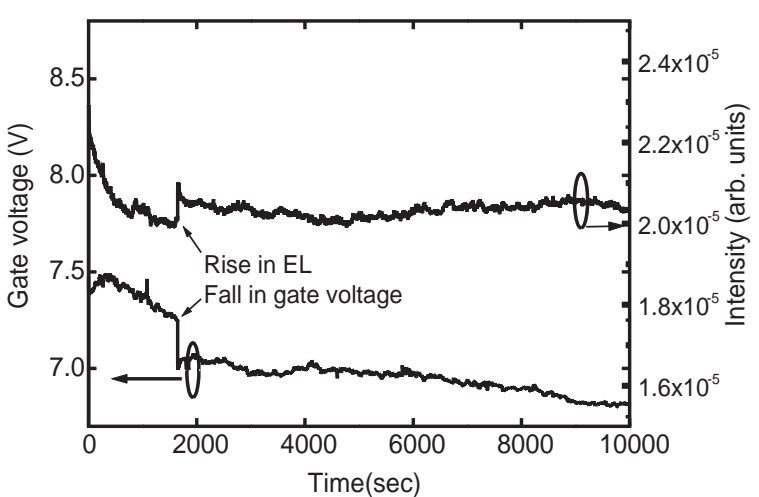

Fig. 4. Reliability test of the MOS diode. Drive current is $100 \mathrm{~mA}$.

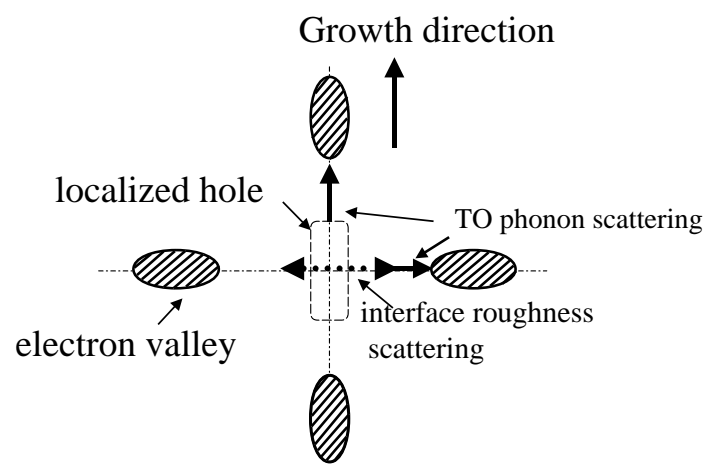

(a)(100)

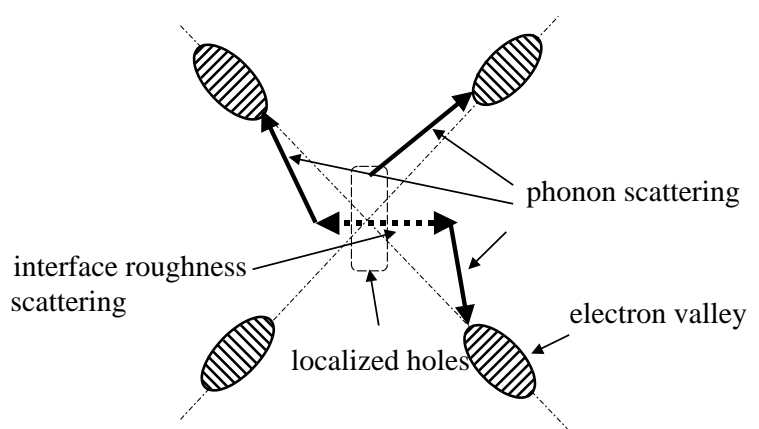

(b)(110)

Fig. 5. A schematic diagram in the reciprocal space to illustrate the radiative recombination in a MOS tunneling diode. Note that the two electron valleys perpendicular to the paper are not shown.

finement in the accumulation region leads to the delocalization of the hole momentum in the reciprocal lattice along the growth direction. The hole in the valence band edge does not have fixed momentum, but has a momentum distribution along the growth direction. In Fig. 5, the momentum associated with the hole is indicated by a rectangle with long sides along the growth direction. To induce hole recombination with electrons, the extra momentum can be provided by phonons. ${ }^{5)}$ Due to the momentum distribution of holes, more phonons can be involved in the radiative recombination, as compared to the bulk $\mathrm{Si}$, where the phonon momentum to assist the radiative recombination is restricted to the reciprocal vector between the valence band edge and electron valley (0.85 $2 \pi / a$, where a is lattice constant). This explains in part why the EL from the MOS diode is more intense than the 
photoluminescence from the bulk Si. The delocalization of the hole momentum can enhance the radiative recombination of holes with electrons in the two electron valleys along the growth direction on (100) substrates, while in the four electron valleys not perpendicular to the growth direction on (110) substrates. The Si/oxide interface roughness can also provide the momentum for the radiative process ${ }^{2,9,10)}$ with the assistance of phonons. The interface roughness also has a distribution of momentum, and more phonons can be involved in the radiative process, as compared to the bulk $\mathrm{Si}$. This is another reason why the EL from the MOS diode is more intense than the photoluminescence from the bulk Si. Due to the two-dimensional distribution of interface roughness, the radiative recombination can be enhanced for the four electron valleys perpendicular to the growth direction on (100) substrates, while for all the six electron valleys on (110) substrates. On the (110) substrates, the four electron valleys not perpendicular to the growth direction can be involved in the enhancement of radiative process by the delocalization of a hole momentum, and all six electron valleys can be involved by the interface roughness. On the (100) substrate, the two electron valleys along the growth direction can be involved in the enhancement of the radiative process by the delocalization of a hole momentum, and the four electron valleys perpendicular to the growth direction can be involved by the interface roughness. Since more electron valleys can be involved in the enhanced radiative process on (110) substrates, as compared to (100) substrates, the (110) substrate has the potential to emit more light.

In conclusion, the EL from an NMOS diode on (110) sub- strates was demonstrated. For the reliability test, the device does not significantly degrade over a 10000 -s operation at $100 \mathrm{~mA}$. A comprehensive picture of the radiation process is described. From this illustration, the EL from the MOS diode on (110) substrates can be more intense than that on the (100) substrates.

This work was supported by the National Science Council, Taiwan, R.O.C. (89-2218-E-002-017, 89-2218-E-002012, 89-2112-M-002-034 and 89-2215-E-002-016). The support from the Tjing Ling Foundation is also highly appreciated.

1) C. W. Liu, M. H. Lee, C. F. Lin, I. C. Lin, W. T. Liu and H. H. Lin: Tech. Dig. Int. Electron Device Meet., 1999 (1999) p. 749.

2) C. W. Liu, M. H. Lee, M.-J. Chen, C.-F. Lin and M. Y. Chern: to be published in IEEE Electron Device Lett. (2000).

3) C. W. Liu, W. T. Liu, M. H. Lee, W. S. Kuo and B. S. Hsu: IEEE Electron Device Lett. 21 (2000) 307.

4) C. W. Liu, M. H. Lee, M.-J. Chen, I. C. Lin and C.-F. Lin: Appl. Phys. Lett. 76 (2000) 1516.

5) C. W. Liu, M.-J. Chen, I. C. Lin, M. H. Lee and C.-F. Lin: Appl. Phys. Lett. 77 (2000) 1111.

6) C. W. Liu, J. C. Sturm, Y. R. Lacroix, M. L. W. Thewalt and D. D. Perovic: Appl. Phys. Lett. 65 (1994) 76.

7) A. Ghetti, E. Sangiorgi, J. Bude, T. W. Sorsch and G. Weber: Tech. Dig. Int. Electron Device Meet., 1999 (1999) p. 731.

8) T. Tomita, H. Utsunomiya, T. Sakura, Y. Kamakura and K. Taniguchi: IEEE Trans. Electron Devices 46 (1999) 159.

9) Y. Uehara, J. Watanabe, S. Fujikawa and S. Ushioda: Phys. Rev. B 51 (1995) 2229.

10) A. Pirovano, A. L. Lacaita, G. Ghidini and G. Tallarida: IEEE Electron Device Lett. 21 (2000) 34. 